\title{
Analytical Cryo Electron Microscopy for Characterization of Pickering Emulsions
}

Teresa Roncal-Herrero $^{1 *}$, Stuart Micklethwaite ${ }^{1}$, Martha Ilett ${ }^{1}$, James Hitchcock ${ }^{1}$, Oliver Cayre $^{1}$ and Nicole Hondow ${ }^{1}$

1. School of Chemical and Process Engineering, University of Leeds, Leeds, United Kingdom.

* Corresponding author: t.roncal@leeds.ac.uk

Pickering emulsions are liquid droplets stabilized by particles located at the liquid/liquid interface [1]. Different types of stabilizers can be used, including inorganic nanoparticles, a combination of metallic nanoparticles and polymers, etc [1]. They are relatively easy to prepare and can be remarkably stable to coalescence and coagulation and compatible with a wide range of biomolecules. As a result, Pickering emulsions have potential applications in numerous different fields such as pharmaceutics, drug delivery, cosmetics, the food industry, catalysis, etc. Conventionally, the characterization of such materials is generally carried out using bulk measurement techniques such as dynamic light scattering, laser diffraction or optical microscopy. The spatial resolution of such methods is limited for these systems, due to the differing physical and chemical characteristics of Pickering emulsions, for example they contain multiple sizes (nominally; oil droplets $50 \mathrm{~nm}-10 \mu \mathrm{m}$, supporting particles $2 \mathrm{~nm}-300 \mathrm{~nm}$ ), which may be non-spherical and contain materials with a low refractive index with respect to the dispersive medium.

These complications in the analysis, combined with a need to understand behavior in order to produce more effective Pickering emulsions, presents the opportunity to use electron microscopy for nanoscale imaging and chemical analysis. To avoid the drying and dehydration effects of conventional sample preparation, we have utilized cryogenic approaches [2] to examine platinum nanoparticle stabilized oil droplets [3]. Samples were analyzed using a FEI Titan Themis ${ }^{3}$ G2 100 transmission electron microscope (TEM) operated at $300 \mathrm{kV}$ with a monochromator, a Super-X 4-detector silicon drift energy dispersive X-ray (EDX) system and a Gatan One view CCD. Scanning TEM (STEM) probe currents were varied by the monochromator and ranged from $10-200 \mathrm{pA}$ depending on the imaging and mapping mode. For dry state observations a $3.5 \mu$ drop of the Pickering emulsion was pipetted onto a $\mathrm{SiN}_{\mathrm{x}}$ grid and air dried. Cryo-TEM sample preparation was carried out using a FEI VitrobotC with a 3.5 $\mu \mathrm{l}$ drop of the Pickering emulsion pipetted onto a lacey carbon coated grid. The grid and sample were blotted and plunged into liquid ethane, then transferred to the TEM using a Gatan 914 TEM cryo-holder. Further analysis has been undertaken using cryo-focused ion beam scanning electron microscopy (FIBSEM), where frozen samples were examined using a Helios G4 CX FIB-SEM following use of a Quorum SEM cryo-transfer unit.

The advantage of using a cryogenic approach is evident when compared to images collected from a dried sample (Figure 1). In the dried sample (Figure 1a) the integrity of the emulsion droplets is not maintained, with most appearing to have burst when drying or due to the vacuum of the microscope. Meanwhile, cryo-STEM shows the emulsion droplets to be spherical with diameters varying from $20 \mathrm{~nm}$ to $2 \mu \mathrm{m}$. Cryo-STEM-EDX mapping shows a carbon-rich oil within the droplet and $\sim 3 \mathrm{~nm} \mathrm{Pt}$ nanoparticles stabilizing the surface (Figure 1b). The size and shape has further been investigated using both cryo-STEM tomography and cryo-FIB-SEM slice and view. Cryo-STEM and FIB-SEM are in this case an indispensable tool for characterization of Pickering emulsions in the native state, minimizing the electron beam damage and drying artefacts [4]. 


\section{References:}

[1] SU Pickering, J. Chem. Soc. Trans. 91 (1907), p. 2001.

[2] M Ilett, R Brydson, A Brown and N Hondow, Micron 120 (2019), p. 35.

[3] JP Hitchcock et al., ACS Appl. Mater. Interfaces 7 (2015), p. 14808.

[4] The authors acknowledge funding from the UK's Engineering and Physical Sciences Research Council under grants EP/R043388/1, EP/M028143/1 and EP/P00122X/1.
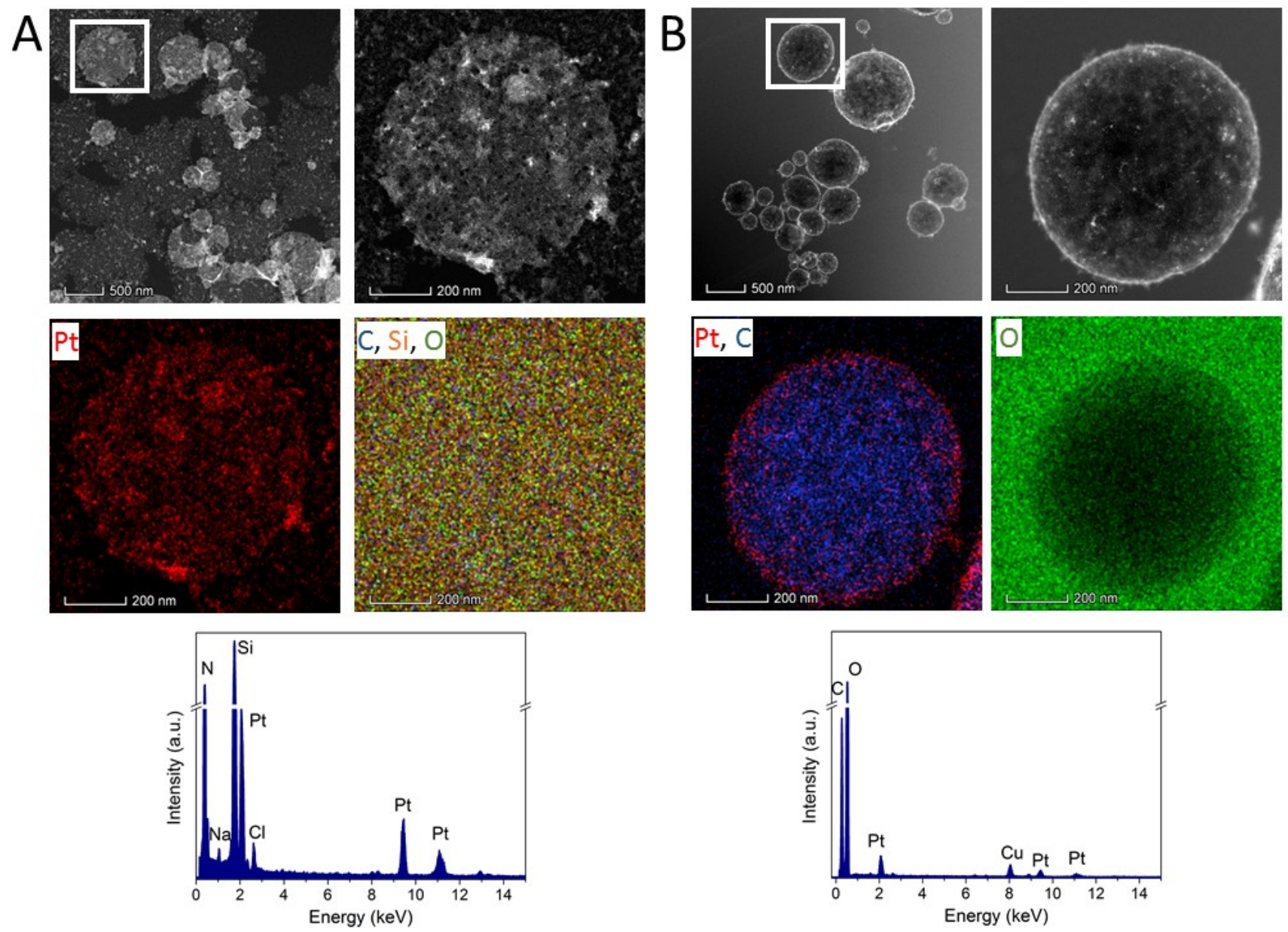

Figure 1. a) HAADF-STEM images of a dropcast Pickering emulsion onto a $\mathrm{SiN}_{\mathrm{x}}$ grid and its corresponding EDX maps and spectrum of the oil droplet b) Cryo-HAADF-STEM images of the frozen Pickering emulsion and its corresponding cryo-EDX maps and spectrum. 